\title{
MicroRNA let-7g alleviates atherosclerosis via the targeting of LOX-1 in vitro and in vivo
}

\author{
MINGXIN LIU ${ }^{1}$, GUIZHOU TAO ${ }^{2}$, QIFENG LIU ${ }^{2}$, KUN LIU ${ }^{1}$ and XINCHUN YANG ${ }^{1}$ \\ ${ }^{1}$ Cardiovascular Center, Beijing Chaoyang Hospital, Capital Medical University, Beijing 100020; \\ ${ }^{2}$ Department of Cardiology, The First Affiliated Hospital of Jinzhou Medical University, Jinzhou, Liaoning 121001, P.R. China
}

Received April 18, 2016; Accepted May 11, 2017

DOI: 10.3892/ijmm.2017.2995

\begin{abstract}
Atherosclerosis is a chronic arterial disease and the leading cause of stroke and myocardial infarction. MicroRNAs (miRNAs or miRs) have been reported to act as essential modulators during the progression of atherosclerosis. Although miR-let-7g has been demonstrated to contribute to maintaining endothelial function and vascular homeostasis, it is not known whether miR-let-7g exerts a therapeutic effect on experimental atherosclerosis. The aim of this study was to investigate the effects of miR-let- $7 \mathrm{~g}$ on atherosclerosis in vivo and in vitro and to explore its underlying mechanisms. Data from our study showed that exogenous lectin-like oxidized low-density lipoprotein receptor-1 (LOX-1 or OLR1) overexpression resulted in the significant promotion of proliferation and migration of human aortic smooth muscle cells (ASMCs), whereas such changes induced by LOX-1 were obviously suppressed by transfection of miR-let-7g. We later confirmed that LOX-1 is a potential target of miR-let-7g, and miR-let-7g markedly inhibited LOX-1 expression in ASMCs by directly binding to the 3 ' untranslated region of LOX-1. Furthermore, in a hyperlipidemic apolipoprotein E knockout $\left(\mathrm{ApoE}^{-/-}\right)$mouse model, intravenous delivery of miR-let- $7 \mathrm{~g}$ mimics obviously attenuated high-fat diet-induced neointima formation and atherosclerotic lesions, accompanied by the significant downregulation of LOX-1, which was consistent with the effect of miR-let-7g on ASMCs. Taken together, our data revealed that miR-let-7g exhibits anti-atherosclerotic activity, at least partially by targeting the LOX-1 signaling pathway. This study suggests that miR-let-7g may be a therapeutic candidate for treating atherosclerosis, and provides novel insight into miRNA-based therapy for this disease.
\end{abstract}

Correspondence to: Professor Xinchun Yang, Cardiovascular Center, Beijing Chaoyang Hospital, Capital Medical University, 8 South Gongti Road, Beijing 100020, P.R. China

E-mail: xinchun_yang0108@tom.com

Key words: apolipoprotein $\mathrm{E}$ knockout mice, $\mathrm{ApoE}^{-/-}$mice, atherosclerosis, lectin-like oxidized low-density lipoprotein receptor-1, microRNA let-7g, aortic smooth muscle cells

\section{Introduction}

Atherosclerosis is a chronic inflammatory disease which is characterized by the formation of atherosclerotic plaques in the arterial wall of large and medium-sized arteries (1). Various pathological events, including proliferation and migration of vascular smooth muscle cells (VSMCs) and endothelial cells (ECs), formation of foam cells, deposition of extracellular lipid, persistent inflammation and oxidative stress, contribute to the progression of atherosclerosis (1-3). Atherosclerosis has been reported to be the primary cause of myocardial infarction, heart failure, myocardial ischemia as well as stroke and accounts for high mortality and morbidity worldwide $(4,5)$. Although many therapeutic medicines for atherosclerosis have been widely applied in the clinic, there are subgroups of patients who remain at high risk of the aforementioned cardiovascular diseases. Therefore, exploring new therapeutic targets or/and developing more effective treatments for atherosclerosis is a top priority.

LOX-1, lectin-like oxidized low-density lipoprotein (oxLDL) receptor (also named as OLR1) expressed in VSMCs, vascular ECs and macrophages, plays a critical role in the pathogenesis of atherosclerosis and has attracted considerable research attention (6,7). Accumulating evidence indicates that oxLDL-induced endothelial dysfunction, VSMC proliferation, and foam cell formation are associated with LOX-1 overexpression $(8,9)$. In addition, it has been shown that LOX-1 is strongly upregulated in atherosclerotic plaques from experimental animals and human atherosclerosis $(10,11)$. Moreover, deletion of LOX-1 was found to lead to a marked reduction in atherosclerotic lesions in LOX-1-knockout mice fed a high cholesterol diet (12). Accordingly, LOX-1 has been suggested as a potential therapeutic target for treating atherosclerosis (5).

MicroRNAs (miRNAs or miRs) are endogenous small non-coding RNAs that can bind to the $3^{\prime}$ untranslated region (3'UTR) of target mRNAs to negatively regulate their expression (13). Increasing evidence implicates specific miRNAs as essential modulators for vascular functions and diseases including atherosclerosis $(14,15)$. Several studies support the notion that miR-145-targeted therapy reduces atherosclerosis in vitro and in vivo and have highlighted the potential application of miRNA-based gene therapy in atherosclerosis (16-18). Therefore, discovering new miRNA-based therapeutic strategies to combat atherosclerosis is warranted. 
miR-let-7g, a key member of the let-7 family which is highly conserved across animal species, has recently attracted considerable attention due to its various biological functions. Several studies indicate that let-7g participates in oxLDL-induced proliferation and apoptosis of ECs and VSMCs (19-21). More recently, Liao et al demonstrated that let-7g contributes to maintaining endothelial function and vascular homeostasis by targeting transforming growth factor- $\beta$ (TGF- $\beta$ ) and SIRT-1 (22). All of these findings motivated us to speculate that the targeting of miR-let-7g may be a potential miRNA-based treatment for atherosclerosis. In the present study, to test this hypothesis, the effects of miR-let- $7 \mathrm{~g}$ on atherosclerosis and its underlying mechanisms were investigated in vitro and in vivo. We demonstrated that the overexpression of miR-let-7g significantly inhibited cell proliferation and migration in LOX-1-overexpressed VSMCs by targeting LOX-1. Moreover, intravenous delivery of miR-let-7g mimics obviously attenuated atherosclerotic lesions and neointima formation as well as downregulated the expression of LOX-1 in a hyperlipidemic apolipoprotein E knockout (ApoE ${ }^{-/}$) mouse model. Our results suggest that miR-let-7g is a valuable therapeutic tool for atherosclerosis, and provides novel insight into miRNA-based therapy for this disease.

\section{Materials and methods}

Cell culture and transfection. Human aortic smooth muscle cells (ASMCs) were purchased from CHI Scientific, Inc. (Jiangsu, China). ASMCs were maintained in Dulbecco's modified Eagle's medium (DMEM; Gibco, Rockville, MD, USA) supplemented with $10 \%$ fetal bovine serum (FBS; Hyclone, Logan, UT, USA) and $1 \%$ penicillin-streptomycin at $37^{\circ} \mathrm{C}$ in a humidified atmosphere with $5 \% \mathrm{CO}_{2}$.

The LOX-1 overexpression vector (pCMV3-LOX-1), pcDNA3.1-let-7g vector, and pcDNA3.1-let-7g-mutant vector were constructed by Wanleibio Co., Ltd. (Shenyang, China). For cell transfection, ASMCs were seeded into cell culture plates or dishes and cultured for $24 \mathrm{~h}$. Following incubation with serum-free medium for $1 \mathrm{~h}$, the cells were transfected with the pCMV3-LOX-1 or pcDNA3.1-let-7g vector, or co-transfected with both vectors using Lipofectamine 2000 (Invitrogen Life Technologies, Carlsbad, CA, USA) according to the manufacturer's instructions. The pcDNA3.1-let-7g-mutant vector served as the negative control for pcDNA3.1-let-7g, while pCMV3 and pcDNA3.1 vectors were used as internal controls. After $5 \mathrm{~h}$ of transfection, the medium was replaced with DMEM containing $10 \%$ FBS, and the cells were used for the subsequent experiments.

Cell proliferation assay. Cell proliferation was detected using the 3-(4,5-dimethylthiazol-2-yl)-2,5-diphenyltetrazolium bromide (MTT) assay. Briefly, ASMCs were seeded in quintuplicate wells of 96-well plates at $1 \times 10^{3}$ cells/well for $24 \mathrm{~h}$, and then transfected with plasmids as described above. At $5 \mathrm{~h}$ of post-transfection, the cell medium was replaced with DMEM containing $10 \%$ FBS and the cells continued to incubate for up to $0,24,48,72$ or $96 \mathrm{~h}$. After that, $20 \mu \mathrm{l}$ MTT solution was added to each well and incubated for $4 \mathrm{~h}$ at $37^{\circ} \mathrm{C}$. Subsequently, the supernatant was discarded and $200 \mu \mathrm{l}$ dimethyl sulfoxide (DMSO) was added to dissolve the formazan crystals.
Finally, absorbance was determined at $490 \mathrm{~nm}$ with a microplate reader (ELX-800; BioTek Instruments, Inc., Winooski, VT, USA).

Wound healing assay. ASMCs were seeded in triplicate wells of 6-well plates for $24 \mathrm{~h}$ and then transfected as described above. When the transfected cells reached $90 \%$ confluence, the cells were deprived of medium and wounded with sterile $200-\mu 1$ pipette tips. After washing with serum-free medium to remove cellular debris, photographic images of the wound area were captured under a phase-contrast microscope (AE31; Motic, Xiamen, China). Subsequently, the cells were incubated with serum-free medium for another $24 \mathrm{~h}$, and the images were captured again. The wound closure was assessed by measuring the horizontal distance of the migrating cells from the initial wound.

Transwell migration assay. After transfection with the indicated plasmids as described above, the cells were detached by $0.25 \%$ trypsin-EDTA and resuspended in serum-free DMEM at a density of $5 \times 10^{4}$ cells $/ \mathrm{ml}$. Then, $0.8 \mathrm{ml}$ medium containing $20 \%$ FBS was added to each bottom well, and $200 \mu \mathrm{l}$ cell suspension was placed in the upper chamber of the Transwell chamber (Corning Inc., Lowell, MA, USA). Following incubation for $24 \mathrm{~h}$ at $37^{\circ} \mathrm{C}$, the cells in the upper chamber were removed by wiping the top surface of the membrane with cotton swabs. Subsequently, the migrated cells that had attached to the under surface were fixed with $4 \%$ paraformaldehyde for $20 \mathrm{~min}$ and stained with $0.5 \%$ crystal violet solution for $5 \mathrm{~min}$. After washing with distilled water, the cells in 5 fields of view from each membrane were counted under a light microscope.

Luciferase reporter assay. ASMCs were seeded in triplicate in 6 -well plates at $2 \times 10^{5}$ cells/well and cultured for $24 \mathrm{~h}$. Following incubation with serum-free medium for $1 \mathrm{~h}$, the cells were transiently co-transfected with pmirGLO or pmirGLO-LOX-1-3'UTR reporter plasmid and pcDNA3.1, pcDNA3.1-let-7g or pcDNA3.1-let-7g-mutant vector using Lipofectamine 2000 according to the manufacturer's instructions. At 24 h post-transfection, the cells were harvested, and luciferase activity was detected using the Dual Luciferase Reporter Assay kit (Promega, Madison, WI, USA) according to the manufacturer's instructions. The data of luciferase activity was normalized to the Renilla luciferase activity.

Animal model and treatment. All animal care and experimental protocols were approved by the Animal Care Ethics and Use Committee of Capital Medical University and performed in accordance with the guidelines of this committee. Eight-week-old male ApoE ${ }^{-/-}$mice were purchased from Vital River Laboratory Animal Technology Co.,Ltd.(Beijing, China). After acclimatization for 1 week, the mice were randomly divided into four groups: the control, the model, the scramble miRNA and miR-let-7g mimic groups. The mice in the control group were fed with a standard rodent diet for 12 weeks. To accelerate the development of spontaneous atherosclerosis in $\mathrm{ApoE}^{-/-}$mice, the animals in the other three groups received a high-fat diet $(0.15 \%$ cholesterol plus $21 \%$ fat $)$ for 12 weeks. Starting from the 13th week, the mice in the miR-let- $7 \mathrm{~g}$ mimics group were intravenously administered $20 \mathrm{mg} / \mathrm{kg}$ miR-let-7g 
Table I. Sequences of the primers used for real-time PCR.

\begin{tabular}{|c|c|c|}
\hline $\begin{array}{l}\text { Gene } \\
\text { names }\end{array}$ & $\begin{array}{l}\text { Primer sequences } \\
\left.\text { (5' to } 3^{\prime}\right)\end{array}$ & $\begin{array}{l}\text { Product } \\
\text { size (bp) }\end{array}$ \\
\hline Homo LOX-1 & $\begin{array}{l}\text { F: GGTCCTTTGCCTGGGATTAG } \\
\text { R: TTCCGAGCAAGGGTTTCTATC }\end{array}$ & 207 \\
\hline Homo $\beta$-actin & $\begin{array}{l}\text { F: CTTAGTTGCGTTACACCCTTTCTTG } \\
\text { R: CTGTCACCTTCACCGTTCCAGTTT }\end{array}$ & 156 \\
\hline Mus LOX-1 & $\begin{array}{l}\text { F: TTCCCTGCTGCTATGACTCT } \\
\text { R: GTAAGGTTCGCTTGGTATTG }\end{array}$ & 119 \\
\hline Mus $\beta$-actin & $\begin{array}{l}\text { F: CTGTGCCCATCTACGAGGGCTAT } \\
\text { R: TTTGATGTCACGCACGATTTCC }\end{array}$ & 155 \\
\hline
\end{tabular}

LOX-1, lectin-like oxidized low-density lipoprotein receptor-1; PCR, polymerase chain reaction; $\mathrm{F}$, forward; $\mathrm{R}$, reverse.

mimics for 3 weeks (twice a week). Meanwhile, each mouse in the scramble microRNA group was given an equal volume of scramble microRNA for 3 weeks. At the end of the treatment, the mice were sacrificed by cervical dislocation under anesthesia, and complete aortas were removed. Some samples were flash-frozen in liquid nitrogen and kept at $-80^{\circ} \mathrm{C}$, whereas the others were fixed in paraformaldehyde.

Hematoxylin and eosin (H\&E) staining assay. After $24 \mathrm{~h}$ of fixation in $4 \%$ paraformaldehyde, the mouse aortas were dehydrated and then embedded in paraffin. Subsequently, paraffin-embedded aortic tissues were cross-sectioned into $5-\mu \mathrm{m}$ sections, dewaxed and rehydrated. Serial sections were stained with H\&E solution following the manufacturer's instructions. Images were captured using an optical microscope (DP73; Olympus, Tokyo, Japan) to estimate vascular morphology and neointimal formation.

Quantitative (real-time) polymerase chain reaction ( $q P C R$ ). Total RNAs were extracted from ASMCs and mouse aortas using the RNApure Rapid Extraction Total kit (BioTeke Corporation, Beijing, China) according to the manufacturer's suggested protocols. cDNAs were produced from equal samples of total RNA by reverse transcription in a reaction system containing random primers and M-MLV reverse transcriptase. Quantitative (real-time) PCR analysis was performed in Exicycler 96 Real-Time Quantitative Thermal Block (Bioneer, Daejeon, Korea). The reaction was initiated by heating at $95^{\circ} \mathrm{C}$ for $10 \mathrm{~min}$, and then 40 cycles of $95^{\circ} \mathrm{C}$ for $10 \mathrm{sec}, 60^{\circ} \mathrm{C}$ for $20 \mathrm{sec}$ and $72^{\circ} \mathrm{C}$ for $30 \mathrm{sec}$, followed by a final incubation at $4^{\circ} \mathrm{C}$ for $5 \mathrm{~min}$. The primers used are shown in Table I. The relative expression of mRNA was normalized to the internal control $\beta$-actin and calculated using the $2^{-\Delta \Delta C T}$ threshold method.

Western blot analysis. Total protein from ASMCs and mouse aortas was extracted using RIPA buffer supplemented with PMSF, and protein concentrations were quantified using the BCA protein assay kit (all from Beyotime Institute of Biotechnology, Jiangsu, China) according to the manufacturer's instructions. Equal amounts of proteins were separated by $11 \%$ SDS-PAGE and subsequently transferred to polyvinylidene difluoride (PVDF) membranes (Millipore, Billerica, MA, USA). The membranes were then blocked with 5\% non-fat milk in Tris-buffered saline with Tween-20 (TBST) for $1 \mathrm{~h}$, followed by incubation with the primary antibodies against LOX-1 (1:500; D160550; Sangon Biotech Co., Ltd., Shanghai, China) and $\beta$-actin (1:1,000; sc-47778; Santa Cruz Biotechnology, Inc., Dallas, TX, USA) overnight at $4^{\circ} \mathrm{C}$. After washing with TBST, the blots were incubated with horseradish peroxidase (HRP)-conjugated secondary antibody $(1: 5,000$; A0208 or A0216; Beyotime Institute of Biotechnology) at $37^{\circ} \mathrm{C}$ for $45 \mathrm{~min}$. Thereafter, the proteins of interest were visualized using enhanced chemiluminescence (ECL; 7Sea Biotech Co., Ltd., Shanghai, China) and densitometric analysis was performed using Gel-Pro Analyzer system (Liuyi Instrument Factory, Beijing, China). The relative expression of protein was calculated by normalization to the internal control $\beta$-actin.

Immunofluorescence assay. The paraffin-embedded aortic tissue sections were dewaxed with xylene, hydrated with gradient ethanol, and microwaved for antigen retrieval for $10 \mathrm{~min}$. After washing with phosphate-buffered saline (PBS), the sections were blocked with goat serum (Solarbio, Beijing, China) at room temperature for $30 \mathrm{~min}$, followed by incubation with the primary antibody against LOX-1 (1:100; D160550; Sangon Biotech Co., Ltd.) in a humidified chamber at $4^{\circ} \mathrm{C}$ overnight. After rinsing with PBS, the slides were incubated with Cy3-labeled goat anti-rabbit IgG (1:200; A0516; Beyotime Institute of Biotechnology) at room temperature for $60 \mathrm{~min}$. Then, nuclear staining was carried out with DAPI (BioSharp, Hefei, China). Thereafter, the slides were mounted with neutral glycerine and observed under a fluorescence microscope (BX53; Olympus).

Statistical analysis. Data are presented as mean \pm standard deviation (SD) from at least three independent experiments. Statistical analyses were performed using one-way analysis of variance (ANOVA) followed by Newman-Keul's test. At P-values $<0.05$, the results were considered statistically significant.

\section{Results}

LOX-1 overexpression induces ASMC proliferation and migration. Previous studies have shown that ox-LDL induces SMC proliferation via LOX-1 (20). In order to examine whether LOX-1 overexpression stimulates SMC proliferation, the pCMV3-LOX-1 vector was transfected into ASMCs, and MTT assay was performed. As expected, LOX-1 transfection significantly induced ASMC proliferation in a time-dependent manner, in particular, after $48 \mathrm{~h}$ of transfection (Fig. 1A). Additionally, we also evaluated the impact of LOX-1 overexpression on ASMC migration, an important event contributing to the development of atherosclerosis. The results of the wound healing and Transwell migration assays showed that LOX-1 overexpression markedly enhanced ASMC migration compared with the non-transfected and pCMV3-transfected ASMCs (Fig. 1B-D). Together, these data indicated that LOX-1 overexpression induced not only ASMC proliferation, but also cell migration. 
A

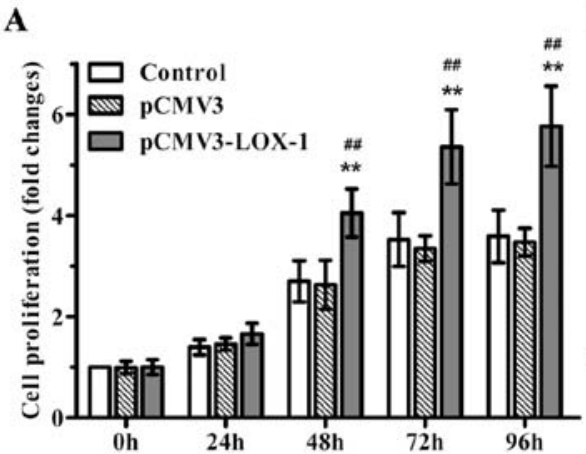

B

Control

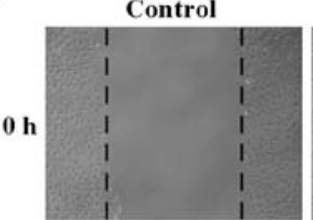

pCMV3

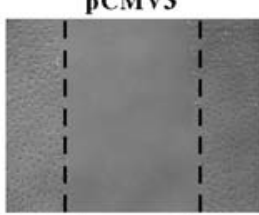

pCMV3-LOX-1

$24 \mathrm{~h}$
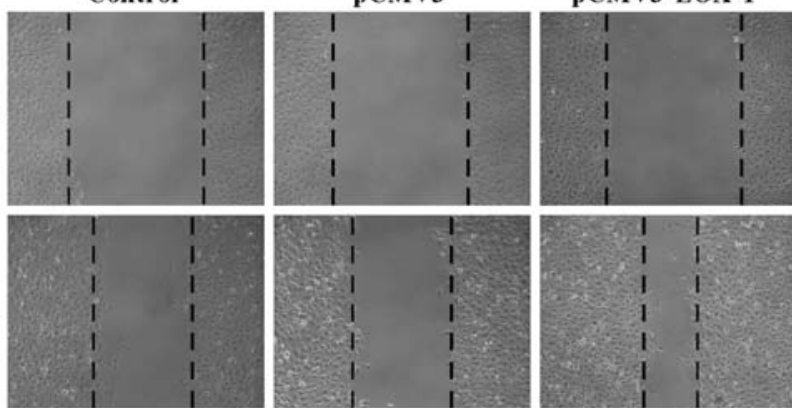

C

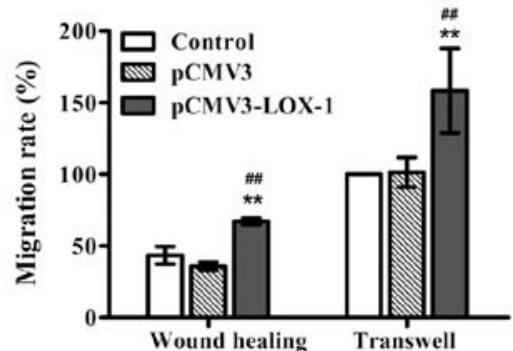

D
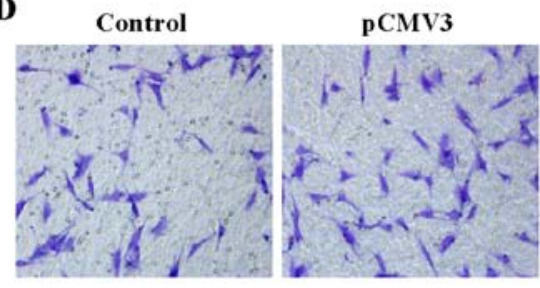

pCMV3-LOX-1

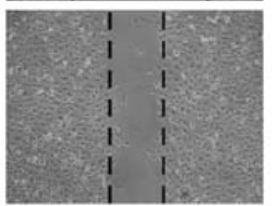

Figure 1. Lectin-like oxidized low-density lipoprotein receptor-1 (LOX-1) overexpression stimulates aortic smooth muscle cell (ASMC) proliferation and migration. ASMCs were transfected with pCMV3-LOX-1 to induce LOX-1 overexpression or pCMV3 vector as a negative control. (A) Cell proliferation was determined using MTT assay at 0,24, 48, 72 and $96 \mathrm{~h}$ post-tranfection. (B) Cell migration was detected using a wound healing assay and (D) Transwell migration assay, and (C) the cell migration rate of these two methods was respectively analyzed as described in Materials and methods. Original magnification was $\mathrm{x} 100$ for (B) and $\mathrm{x} 200$ for (D). Data are presented as mean $\pm \mathrm{SD}(\mathrm{n}=3)$. ${ }^{* *} \mathrm{p}<0.01$ vs. the control; ${ }^{\# \#} \mathrm{p}<0.01$ vs. the pCMV3 vector group.

A

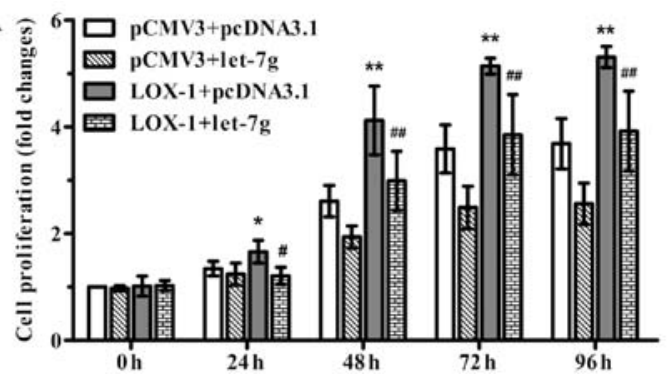

B

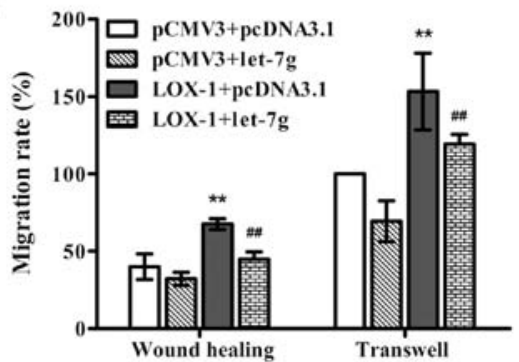

C
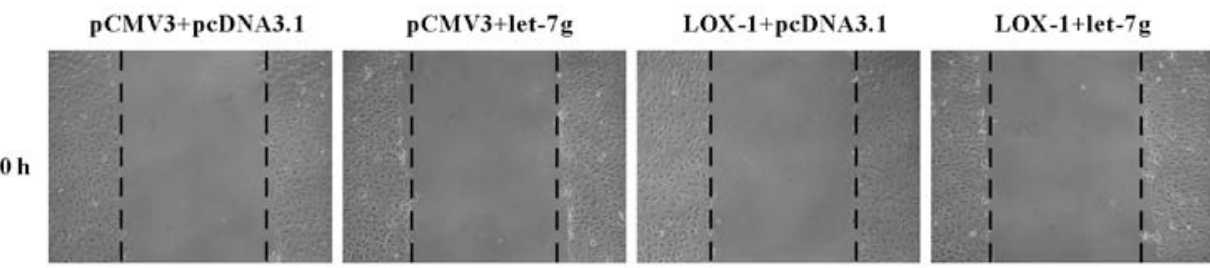

$24 \mathrm{~h}$
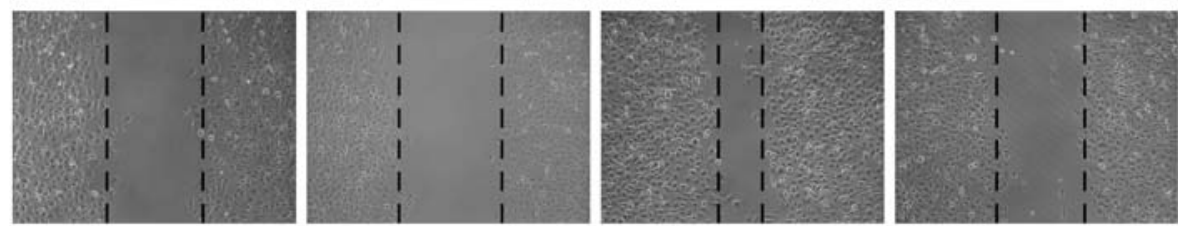

D
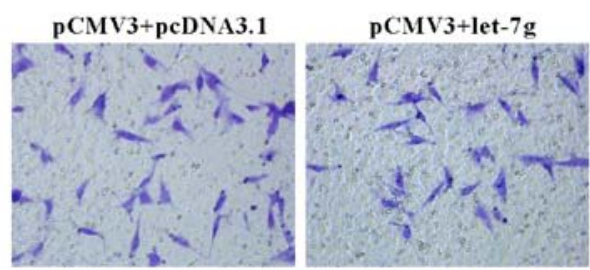

LOX-1+pcDNA3.1

LOX-1+let-7g
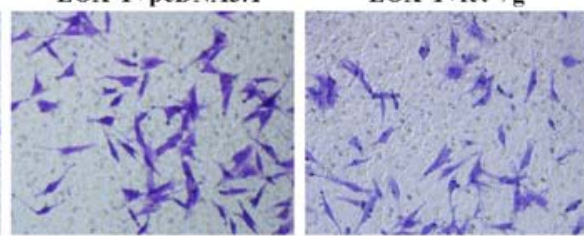

Figure 2. miR-let-7g attenuates lectin-like oxidized low-density lipoprotein receptor-1 (LOX-1)-induced aortic smooth muscle cell (ASMC) proliferation and migration. ASMCs were co-transfected with pCMV3-LOX-1 or pCMV3 vector and pcDNA3.1-let-7g or pcDNA3.1 vector for overexpression of LOX-1 or let-7g or both. (A) Cell proliferation was determined using the MTT assay at $0,24,48,72$ and $96 \mathrm{~h}$ post-tranfection. (C) Cell migration was detected using a wound healing assay and (D) Transwell migration assay, and (B) the ratio of cell migration was analyzed. Original magnification was x100 for (C) and x200 for (D). Data are presented as mean $\pm \mathrm{SD}(\mathrm{n}=3) .{ }^{*} \mathrm{p}<0.05,{ }^{* *} \mathrm{p}<0.01$ vs. pCMV3+pcDNA3.1; ${ }^{*} \mathrm{p}<0.05,{ }^{\# \#} \mathrm{p}<0.01$ vs. pCMV3-LOX-1+pcDNA3.1. 
A

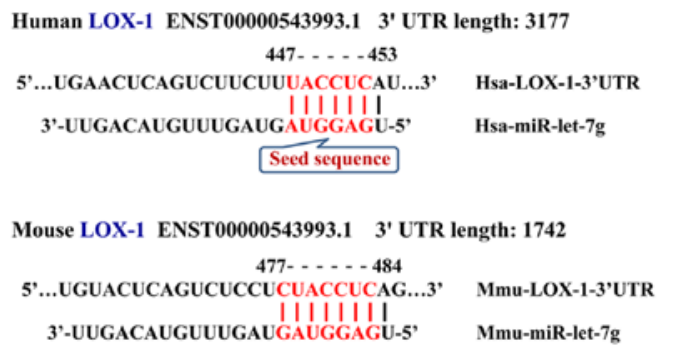

C

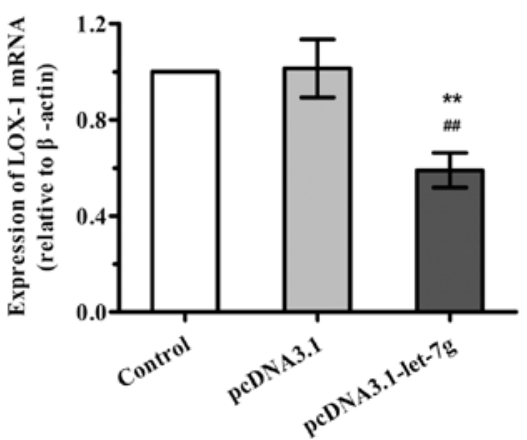

B

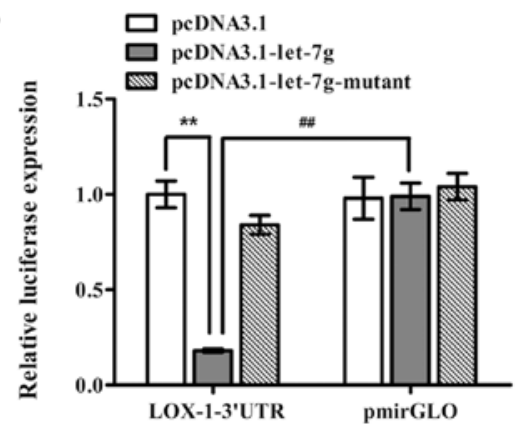

D
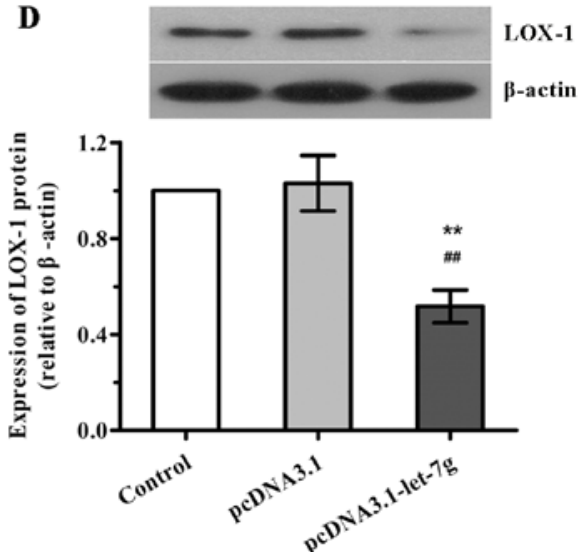

Figure 3. miR-let-7g inhibits lectin-like oxidized low-density lipoprotein receptor-1 (LOX-1) expression in human aortic smooth muscle cells (ASMCs) by binding to its $3^{\prime}$ untranslated region (3'UTR). (A) A sequence complementary to the seed sequence of let-7g was observed in the 3'UTR of LOX-1 mRNA in the human (Hsa-) and mouse (Mmu-). (B) ASMCs were co-transfected with pmirGLO-LOX-1-3'UTR luciferase reporter plasmid and pcDNA3.1-let-7g, mutant or pcDNA3.1 plasmid. At $24 \mathrm{~h}$ post-transfection, luciferase assay was performed. A reporter containing not LOX-1-3'UTR but pmirGLO was used as a negative control. (C and D) ASMCs were transfected with pcDNA3.1-let-7g or its control plasmid, and then (C) the expression levels of LOX-1 mRNA and (D) LOX-1 protein were determined using real-time PCR and western blot analysis, respectively. $\beta$-actin served as the internal control. Data are presented as mean $\pm \mathrm{SD}(\mathrm{n}=3) .{ }^{* *} \mathrm{p}<0.01$ vs. the control; ${ }^{\# \#} \mathrm{p}<0.01$ vs. the pcDNA3.1.

miR-let-7g attenuates LOX-1-induced ASMC proliferation and migration. In order to test the effect of miR-let- $7 \mathrm{~g}$ on LOX-1-induced ASMC proliferation and migration, pCMV3-LOX-1 plasmid and pcDNA3.1-let-7g plasmid were co-transfected into the ASMCs. As shown in Fig. 2A, miR-let$7 \mathrm{~g}$ overexpression markedly suppressed the LOX-1-stimulated proliferation of ASMCs compared to the cells co-transfected with the pCMV3-LOX-1 and scramble pcDNA3.1 vectors as determined by MTT assay. To confirm the inhibitory effect of miR-let-7g on ASMC migration, wound healing and Transwell migration assays were performed. Compared with the ASMCs co-transfected with pCMV3-LOX-1 and scramble pcDNA3.1 plasmids, the number of migratory cells harboring the pCMV3-LOX-1 plasmid and pcDNA3.1-let-7g plasmid were obviously decreased (Fig. 2B-D). These results indicated that miR-let-7g reversed the ASMC proliferation and migration mediated by LOX-1 overexpression.

miR-let-7g inhibits LOX-1 expression in ASMCs by binding to its 3'UTR. Given that miR-let-7g is predicted to bind to the 3'UTR of LOX mRNA in the human and mouse according to TargetScan program and miRNA target prediction database (Fig. 3A), we determined whether miR-let-7g directly binds to the 3'UTR sequence of LOX mRNA and affects its expression in human ASMCs. Firstly, a luciferase reporter vector was constructed with the 3'UTR sequence of LOX-1 containing the putative binding site for miR-let-7g, and lucif- erase report assay was performed. As shown in Fig. 3B, when LOX-1 3'UTR and pcDNA3.1-let-7g were co-tranfected into the ASMCs, the luciferase activity was significantly decreased compared with the scramble pcDNA3.1. Nevertheless, co-transfection of LOX-1 3'UTR and miR-let-7g-mutant as well as co-transfection of scramble pmirGLO and pcDNA3.1let-7g both had no effects on the luciferase activities. Subsequently, to further confirm the functional interaction of miR-let-7g with LOX-1, the effects of miR-let-7g on the expression levels of LOX-1 mRNA and protein in human ASMCs were determined by qPCR and western blot analysis, respectively. As expected, overexpression of miR-let-7g led to a marked reduction in both LOX-1 mRNA and protein expression (Fig. 3C and D), which was consistent with the results of the luciferase reporter activity. Taken together, our findings suggest that miR-let-7g directly targets the 3'UTR of LOX-1 to suppress LOX-1 expression.

miR-let-7g alleviates atherosclerotic lesions in ApoE ${ }^{-/-}$mice. To further evaluate the potential therapeutic benefit of miR-let-7g in atherosclerosis, high-fat diet fed $\mathrm{ApoE}^{-/-}$mice were treated with miR-let-7g mimics. As indicated in Fig. 4A, compared to the mice fed a normal diet, $\mathrm{ApoE}^{-/-}$mice fed a high-fat diet for 12 weeks showed significant neointimal hyperplasia. Importantly, miR-let-7g mimics obviously reduced the neointimal hyperplasia compared with that noted in the scramble-treated aortas. Moreover, the quantification 
$\mathbf{A}$
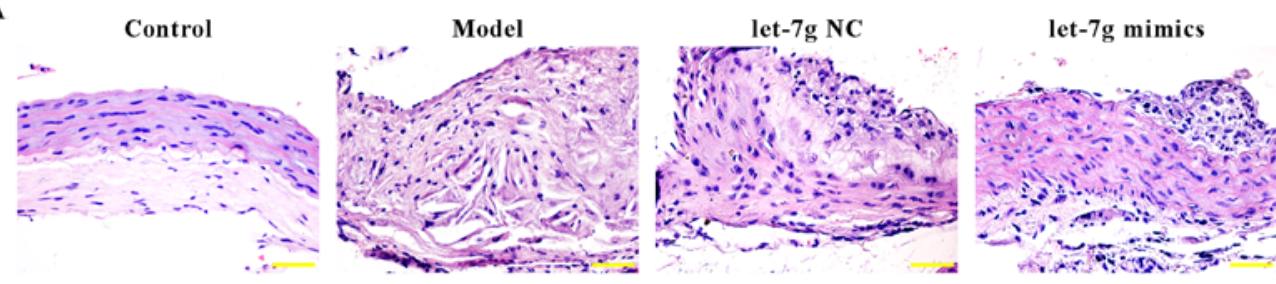

B

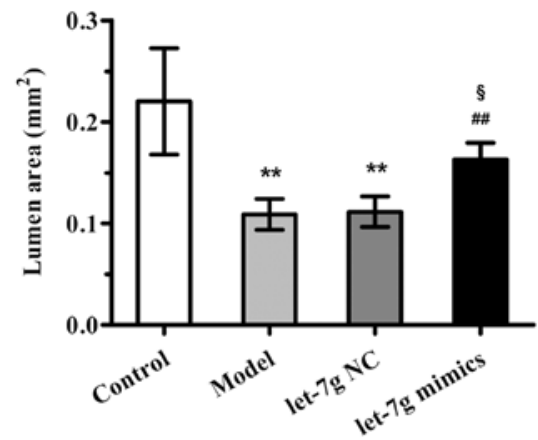

C

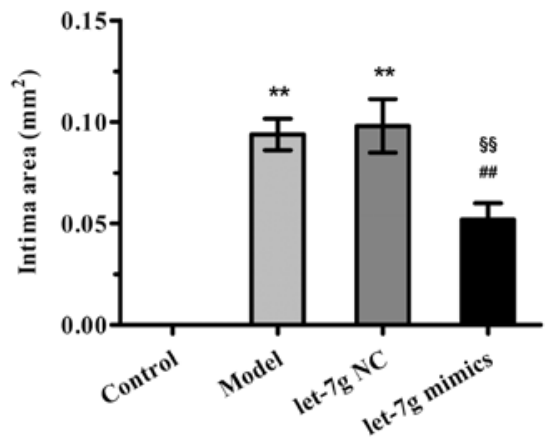

D

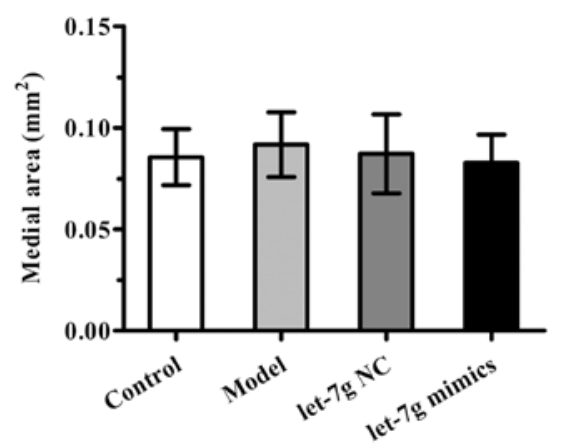

$\mathbf{E}$

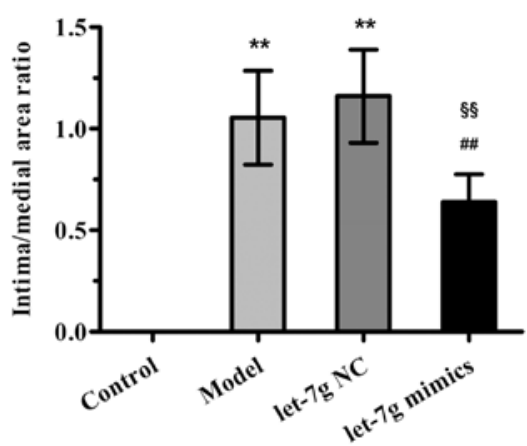

Figure 4. miR-let-7g alleviates atherosclerotic lesions in apolipoprotein E (ApoE ${ }^{-/}$) mice. (A) Aortic tissue sections from each group were stained with hematoxylin and eosin (H\&E) for histological assessment. Representative images are shown. Original magnification was x400. (B) Lumen area, (C) intima area and (D) medial area of the aortas from each group were analyzed by Image-Pro Plus 6.0. (E) The ratio of intima area to medial area was calculated. Data are presented as mean $\pm \mathrm{SD}(\mathrm{n}=6) .{ }^{* *} \mathrm{p}<0.01$ vs. the control group; ${ }^{\# \#} \mathrm{p}<0.01$ vs. the model group; ${ }^{\S} \mathrm{p}<0.05$ and ${ }^{\S \S} \mathrm{p}<0.01$ vs. the let-7g NC group.

results of morphometric analyses revealed that the parameters for severity of atherosclerosis, such as lumen area, intima area (Fig. 4B and C), and intima to media area ratio (Fig. 4E), were markedly improved by miR-let-7g mimic delivery. However, there was no significant difference in medial area (Fig. 4D). These data suggest that miR-let-7g exterts potent anti-atherosclerotic effects in vivo.

miR-let-7g downregulates the expression of LOX-1 in the aortas of $\mathrm{ApoE}^{-/-}$mice. Further experiments were undertaken to demonstrate the underlying mechanisms of interaction between miR-let-7g and LOX-1 in vivo. The results of qPCR and western blot analysis showed that the mRNA and protein expression levels of LOX-1 in the mouse aortas were both notably upregulated in the high-fat fed and NC-treated groups compared with the control animals, with no significant difference observed between the model and NC-treated groups (Fig. 5A and B). By contrast, a marked reduction in LOX-1 expression in the aortas was found after the administration of miR-let-7g mimics. Additionally, concordant with these results, immunofluorescence analysis also confirmed that treatment with miR-let-7g mimics obviously reversed the upregulation of LOX-1 expression in the aortas of the high-fat diet fed $\mathrm{ApoE}^{-/-}$mice. These in vivo observations were consistent with the effects of miR- let-7g on ASMCs, suggesting that miR-let-7g may alleviate atherosclerosis via inhibition of LOX-1 expression.

\section{Discussion}

Currently, the incidence of atherosclerosis is rapidly increasing in developing and developed countries (23). Increasing interest in the treatment of atherosclerosis is currently being focused on miRNAs due to their essential regulatory roles in the development of atherosclerosis (24). In the present study, we investigated the anti-atherosclerotic effect of miR-let- $7 \mathrm{~g}$ both in vitro and in vivo. The data presented here showed that exogenous LOX-1 overexpression had a promoting effects on ASMC proliferation and migration, whereas co-transfection with miR-let-7g into ASMCs reversed these effects. The underlying mechanisms of miR-let-7g likely involve repression of LOX-1 by directly targeting its 3'UTR. Furthermore, the in vivo studies showed that systematic delivery of miR-let-7g attenuated neointima formation in high-fat diet fed $\mathrm{ApoE}^{-/-}$mice, a well-established animal model for studying atherosclerosis. The anti-atherosclerotic effects of miR-let-7g in mice were accompanied by a significant downregulation of LOX-1, consistent with its effects on ASMCs.

During the atherosclerotic process, VSMCs undergo a variety of pathological changes. The balance between differ- 
A

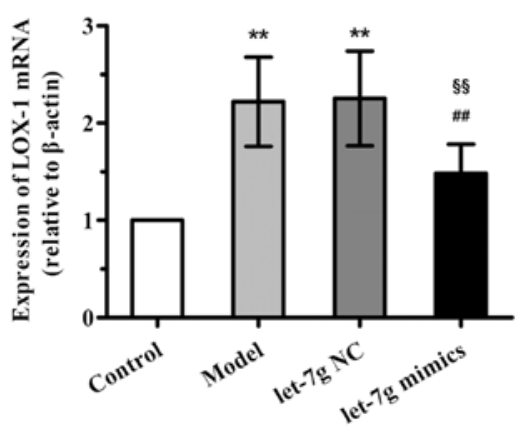

C
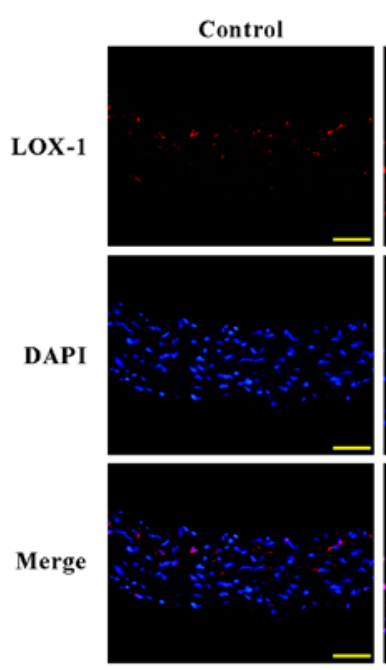

B
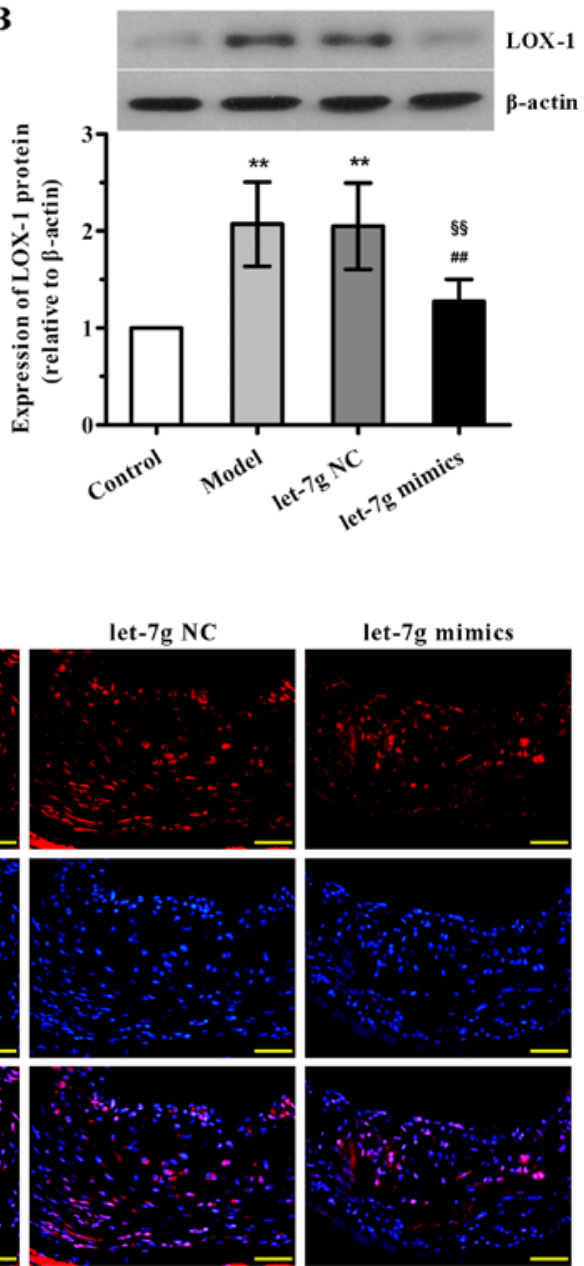

Figure 5. miR-let-7g downregulates the expression of lectin-like oxidized low-density lipoprotein receptor-1 (LOX-1) in the aortas of apolipoprotein E knockout $\left(\mathrm{ApoE}^{-/}\right)$mice. (A) The expression levels of LOX-1 mRNA and (B) LOX-1 protein in the arteries of C57BL/6 mice and ApoE ${ }^{-/-}$mice were evaluated using real-time PCR and western blot analysis, respectively. $\beta$-actin was used as the internal control. Data are presented as mean \pm SD $(n=6)$. ${ }^{*}$ p $<0.01$ vs. the control group; ${ }^{\# \#} \mathrm{p}<0.01 \mathrm{vs}$. the model group; ${ }^{\S \S} \mathrm{p}<0.01 \mathrm{vs}$. the let-7g NC group. (C) Immunofluorescence staining was performed to detect the protein expression of LOX-1 (red) in the arterial sections from each group. Nuclei were visualized by DAPI staining (blue). The representative images of each group are shown. Original magnification was $\mathrm{x} 400$.

entiation and proliferation of VSMCs plays a critical role in maintaining healthy blood vessels. However, some stimulation such as mitogenic substances, growth factors, or loss of vascular ECs can induce VSMC migration from the media into the intima. Subsequently, VSMC proliferation can occur in the intima, leading to neointimal hyperplasia as well as plaque formation (25). Hence, the migration and proliferation of VSMC is the most common pathological alteration in the development of atherosclerosis. Ox-LDL plays a key role in the genesis and progression of atherosclerosis by upregulating its own receptor, LOX-1, which appears to be the dominant receptor on VSMCs and ECs (26). Furthermore, the activation of LOX-1 has also been reported to be involved in many pathological events of atherosclerosis, such as vascular cell apoptosis, proliferation as well as vascular remodeling $(27,28)$. In the present study, we constructed LOX-1-overexpressing ASMCs by transfecting a plasmid containing the full length cDNA of LOX-1 into human ASMCs, and confirmed that exogenous LOX-1 overexpression could significantly induce cell migration and proliferation, suggesting an in vitro model for the development of atherosclerosis.
The miR-let-7 family, consisting of nine members, was identified in humans and has been shown to play a pivotal role in developmental processes (29). More recently, the functions of let-7 in cardiovascular biology and disease have drawn increasing attention (30). It has been found that levels of let-7 are closely associated with coronary artery disease including atherosclerosis. Among let-7 members, miR-let-7g has been demonstrated to improve multiple endothelial functions including an increase in angiogenesis as well as a decrease in monocyte adhesion, inflammation and senescence by targeting TGF- $\beta$ and SIRT-1 signaling pathway (22). Moreover, it has been reported that ox-LDL at lower concentrations causes VSMC proliferation $(<20 \mu \mathrm{g} / \mathrm{ml})$ while it inducs cell apoptosis at higher concentrations $(>60 \mu \mathrm{g} / \mathrm{ml})$, which could be suppressed by miR-let-7g mimic and exacerbated by its inhibitor (20). In addition, a previous study also revealed that miR-let-7g could function as an important modulator of autophagy and apoptosis in ox-LDL-treated VSMCs (27). In this study, we demonstrated that when miR-let-7g was co-transfected with LOX-1 into ASMCs, cell migration and proliferation stimulated by LOX-1 overexpression alone were obviously inhibited, 
which was accompanied by a decrease in the mRNA and protein levels of LOX-1. The reporter assay further confirmed the direct binding of miR-let-7g to the 3'UTR of LOX-1. These observations suggest that miR-let-7g can interfere with ASMC proliferation and migration by targeting LOX-1.

Next, we studied the anti-atherosclerotic effect of miR-let-7g in $\mathrm{ApoE}^{-/-}$mice fed a high-fat diet. We demonstrated that the LOX-1 expression in aortas of the high-fat diet fed ApoE - $^{-/}$mice was markedly increased, consistent with previous studies supporting the notion that LOX-1 accumulates in atherosclerotic lesions of experimental animals and humans $(5,31)$. It has been reported that deletion of LOX-1 attenuates atherosclerosis in LOX-1-knockout mice fed a high cholesterol diet (12), therefore highlighting the key role of LOX-1 in atherosclerosis. Of note, here, our data showed that administration of miR-let-7g mimics markedly downregulated the mRNA and protein levels of LOX-1 as well as ameliorated neointima formation and atherosclerotic lesions, in line with the in vitro findings.

In conclusion, our results demonstrate that miR-let-7g can suppress ASMC proliferation and migration in vitro and alleviate atherosclerosis in $\mathrm{ApoE}^{-/-}$mice, at least partly by directly inhibiting LOX-1 activation. These observations suggest that miR-let-7g may serve as a potential therapeutic strategy for treating atherosclerosis.

\section{Acknowledgements}

This study was supported by a grant from the National Undergraduate Innovative Training Program (no. 201610160005).

\section{References}

1. Libby P: Inflammation in atherosclerosis. Nature 420: $868-874,2002$.

2. Stocker R and Keaney JF Jr: Role of oxidative modifications in atherosclerosis. Physiol Rev 84: 1381-1478, 2004.

3. Obikane H, Abiko Y, Ueno H, Kusumi Y, Esumi M and Mitsumata M: Effect of endothelial cell proliferation on atherogenesis: a role of $\mathrm{p} 21(\mathrm{Sdi} / \mathrm{Cip} / \mathrm{Waf} 1)$ in monocyte adhesion to endothelial cells. Atherosclerosis 212: 116-122, 2010.

4. Zheng Y, Gardner SE and Clarke MC: Cell death, damage-associated molecular patterns, and sterile inflammation in cardiovascular disease. Arterioscler Thromb Vasc Biol 31: 2781-2786, 2011.

5. Liu Z, Xu S, Huang X, Wang J, Gao S, Li H, Zhou C, Ye J, Chen S, Jin ZG, et al: Cryptotanshinone, an orally bioactive herbal compound from Danshen, attenuates atherosclerosis in apolipoprotein E-deficient mice: role of lectin-like oxidized LDL receptor-1 (LOX-1). Br J Pharmacol 172: 5661-5675, 2015.

6. White SJ, Sala-Newby GB and Newby AC: Overexpression of scavenger receptor LOX-1 in endothelial cells promotes atherogenesis in the ApoE(-/-) mouse model. Cardiovasc Pathol 20: 369-373, 2011.

7. Ulrich-Merzenich $\mathrm{G}$ and Zeitler $\mathrm{H}$ : The lectin-like oxidized low-density lipoprotein receptor-1 as therapeutic target for atherosclerosis, inflammatory conditions and longevity. Expert Opin Ther Targets 17: 905-919, 2013.

8. Li D, Chen H, Romeo F, Sawamura T, Saldeen T and Mehta JL: Statins modulate oxidized low-density lipoprotein-mediated adhesion molecule expression in human coronary artery endothelial cells: role of LOX-1. J Pharmacol Exp Ther 302: 601-605, 2002.

9. Mitra S, Goyal T and Mehta JL: Oxidized LDL, LOX-1 and atherosclerosis. Cardiovasc Drugs Ther 25: 419-429, 2011.

10. Draude G, Hrboticky N and Lorenz RL: The expression of the lectin-like oxidized low-density lipoprotein receptor (LOX-1) on human vascular smooth muscle cells and monocytes and its down-regulation by lovastatin. Biochem Pharmacol 57: 383-386, 1999.
11. Chen H, Li D, Sawamura T, Inoue $K$ and Mehta JL: Upregulation of LOX-1 expression in aorta of hypercholesterolemic rabbits: modulation by losartan. Biochem Biophys Res Commun 276: 1100-1104, 2000.

12. Mehta JL, Sanada N, Hu CP, Chen J, Dandapat A, Sugawara F, Satoh H, Inoue K, Kawase Y, Jishage K, et al: Deletion of LOX-1 reduces atherogenesis in LDLR knockout mice fed high cholesterol diet. Circ Res 100: 1634-1642, 2007.

13. Lewis BP, Burge CB and Bartel DP: Conserved seed pairing, often flanked by adenosines, indicates that thousands of human genes are microRNA targets. Cell 120: 15-20, 2005.

14. McDonald RA, Hata A, MacLean MR, Morrell NW and Baker AH: MicroRNA and vascular remodelling in acute vascular injury and pulmonary vascular remodelling. Cardiovasc Res 93: 594-604, 2012.

15. Madrigal-Matute J, Rotllan N, Aranda JF and FernándezHernando C: MicroRNAs and atherosclerosis. Curr Atheroscler Rep 15: 322, 2013.

16. Cheng Y, Liu X, Yang J, Lin Y, Xu DZ, Lu Q, Deitch EA, Huo Y, Delphin ES and Zhang C: MicroRNA-145, a novel smooth muscle cell phenotypic marker and modulator, controls vascular neointimal lesion formation. Circ Res 105: 158-166, 2009.

17. Lovren F, Pan Y, Quan A, Singh KK, Shukla PC, Gupta N, Steer BM, Ingram AJ, Gupta M, Al-Omran M, et al: MicroRNA-145 targeted therapy reduces atherosclerosis. Circulation 126: S81-S90, 2012.

18. Elia L, Quintavalle M, Zhang J, Contu R, Cossu L, Latronico MV, Peterson KL, Indolfi C, Catalucci D, Chen J, et al: The knockout of miR-143 and -145 alters smooth muscle cell maintenance and vascular homeostasis in mice: correlates with human disease. Cell Death Differ 16: 1590-1598, 2009.

19. Zhang Y, Chen N, Zhang J and Tong Y: Hsa-let-7g miRNA targets caspase- 3 and inhibits the apoptosis induced by ox-LDL in endothelial cells. Int J Mol Sci 14: 22708-22720, 2013.

20. Ding Z, Wang X, Khaidakov M, Liu S and Mehta JL: MicroRNA hsa-let-7g targets lectin-like oxidized low-density lipoprotein receptor-1 expression and inhibits apoptosis in human smooth muscle cells. Exp Biol Med (Maywood) 237: 1093-1100, 2012.

21. Chen KC, Hsieh IC, Hsi E, Wang YS, Dai CY, Chou WW and Juo SH: Negative feedback regulation between microRNA let-7g and the oxLDL receptor LOX-1. J Cell Sci 124: 4115-4124, 2011.

22. Liao YC, Wang YS, Guo YC, Lin WL, Chang MH and Juo SH: Let-7g improves multiple endothelial functions through targeting transforming growth factor-beta and SIRT-1 signaling. J Am Coll Cardiol 63: 1685-1694, 2014.

23. Bild DE, McClelland R, Kaufman JD, Blumenthal R, Burke GL, Carr JJ, Post WS, Register TC, Shea S and Szklo M: Ten-year trends in coronary calcification in individuals without clinical cardiovascular disease in the multi-ethnic study of atherosclerosis. PLoS One 9: e94916, 2014.

24. Menghini R, Stöhr R and Federici M: MicroRNAs in vascular aging and atherosclerosis. Ageing Res Rev 17: 68-78, 2014.

25. Libby P, Ridker PM and Hansson GK: Progress and challenges in translating the biology of atherosclerosis. Nature 473: 317-325, 2011.

26. Lu J and Mehta JL: LOX-1: a critical player in the genesis and progression of myocardial ischemia. Cardiovasc Drugs Ther 25: 431-440, 2011.

27. Ding Z, Wang X, Schnackenberg L, Khaidakov M, Liu S, Singla S, Dai Y and Mehta JL: Regulation of autophagy and apoptosis in response to ox-LDL in vascular smooth muscle cells, and the modulatory effects of the microRNA hsa-let-7g. Int J Cardiol 168: 1378-1385, 2013.

28. Ding Z, Liu S, Yang B, Fan Y and Deng X: Effect of oxidized low-density lipoprotein concentration polarization on human smooth muscle cells' proliferation, cycle, apoptosis and oxidized low-density lipoprotein uptake. J R Soc Interface 9: 1233-1240, 2012.

29. Boyerinas B, Park SM, Hau A, Murmann AE and Peter ME: The role of let-7 in cell differentiation and cancer. Endocr Relat Cancer 17: F19-F36, 2010.

30. Bao MH, Feng X, Zhang YW, Lou XY, Cheng Y and Zhou HH: Let-7 in cardiovascular diseases, heart development and cardiovascular differentiation from stem cells. Int J Mol Sci 14: 23086-23102, 2013.

31. Sankaralingam S, Xu Y, Sawamura T and Davidge ST: Increased lectin-like oxidized low-density lipoprotein receptor-1 expression in the maternal vasculature of women with preeclampsia: role for peroxynitrite. Hypertension 53: 270-277, 2009. 